\title{
Optical-phonon-induced frictional drag in coupled two-dimensional electron gases
}

\author{
Hu, Ben Yu-Kuang
}

Published in:

Physical Review B

Link to article, DOI:

10.1103/PhysRevB.57.12345

Publication date:

1998

Document Version

Publisher's PDF, also known as Version of record

Link back to DTU Orbit

Citation (APA):

Hu, B. Y-K. (1998). Optical-phonon-induced frictional drag in coupled two-dimensional electron gases. Physical Review B, 57(19), 12345-12349. https://doi.org/10.1103/PhysRevB.57.12345

\section{General rights}

Copyright and moral rights for the publications made accessible in the public portal are retained by the authors and/or other copyright owners and it is a condition of accessing publications that users recognise and abide by the legal requirements associated with these rights.

- Users may download and print one copy of any publication from the public portal for the purpose of private study or research.

- You may not further distribute the material or use it for any profit-making activity or commercial gain

- You may freely distribute the URL identifying the publication in the public portal

If you believe that this document breaches copyright please contact us providing details, and we will remove access to the work immediately and investigate your claim. 


\title{
Optical-phonon-induced frictional drag in coupled two-dimensional electron gases
}

\author{
Ben Yu-Kuang $\mathrm{Hu}$ \\ Mikroelektronik Centret, Bygning 345b, Danmarks Tekniske Universitet, DK-2800 Lyngby, Denmark
}

(Received 30 July 1997)

\begin{abstract}
The role of optical phonons in frictional drag between two adjacent but electrically isolated two-dimensional electron gases is investigated. Since the optical phonons in III-V materials have a considerably larger coupling to electrons than acoustic phonons (which are the dominant drag mechanism at low $T$ and large separations), it might be expected that the optical phonons will contribute a large effect at high temperatures. The two key differences between optical- and acoustic-phonon-mediated drag are (i) the optical-phonon-mediated interlayer interaction is short-ranged due to the negligible group velocity at the Brillouin zone center, and (ii) the typical momentum transfer for an optical-phonon-mediated scattering is relatively large. These considerations make optical-phonon-mediated drag difficult to see in single-subband GaAs systems, but it may be possible to see the effect in double-subband GaAs systems or single-subband quantum wells in a material with a lower effective mass and lower optical-phonon energy, such as InSb. [S0163-1829(98)05219-9]
\end{abstract}

\section{INTRODUCTION}

Two-dimensional electron gases (2DEGs) in semiconductor quantum wells provide convenient laboratories for the study of interparticle interactions. Recently, it has been possible to make a direct study of these interactions in 2DEGs, based on a proposal made some time ago. ${ }^{1,2}$ Experimentalists have been able to fabricate electrically isolated quantum wells that are only several hundred angstroms apart. Thus, the electrons in the adjacent wells are coupled (through Coulomb and phonon-mediated forces), but are unable to tunnel from one side to the other. These remarkable structures allow the experimentalist to probe directly the interlayer interactions by doing a "simple" transport measurement, i.e., turning on a current in one layer, and measuring the voltage built up in the second. ${ }^{3-9}$ These experiments provide a unique opportunity for a transport measurement to study effects of interparticle interaction.

The most obvious interlayer interaction is of course the Coulomb coupling, which gives relatively featureless dependences (at zero magnetic field) as a function of temperature, relative density, etc. ${ }^{10}$ Experiments, however, have shown that there are interesting pronounced features in drag that are associated with the collective modes in the system, indicating that under the right conditions drag is particularly sensitive to the presence of these modes. So far, the drag effects from both acoustic phonons ${ }^{4,6}$ and plasmons ${ }^{9}$ have been observed and studied theoretically. ${ }^{11-16}$ Güven and Tanatar ${ }^{17}$ studied the effect of optical phonons on plasmon-mediated drag. However, we are unaware of any work on direct optical-phonon-mediated drag.

Looking first at acoustic phonons in drag, it may seem surprising that they can contribute so strongly to the drag transresistivity, given their very weak coupling to the electron gas (relative to the Coulomb interaction). It turns out that strong phase-space effects at particular energy and momentum transfers cause the acoustic phonons to dominate in spite of the weak coupling. ${ }^{16}$ An obvious question arises: if the weak acoustic phonons give such a large contribution, can the optical phonons with a much larger coupling (witness the fact that scattering from optical phonons produces a much larger resistivity than scattering off their acoustic counterparts) give a correspondingly giant drag rate when they come in at higher temperatures?

The answer depends on the type of system one is studying. There are two significant differences between acoustic and optical phonons. The first is that, unlike their acoustic counterparts, the optical phonons around the Brillouin-zone center (those that are relevant for drag) have very small group velocities. Thus, the range of the optical phonons is much shorter than the acoustic phonons. In fact, the longitudinal polar optical-phonon-mediated interaction has spatially exactly the same short-ranged form as the out-of-plane Coulomb interaction in two-dimensional structures; i.e., it is proportional to $\exp (-q d)$, where $\hbar q$ is the electron momentum transferred and $d$ is the center-to-center interlayer separation. Furthermore, every optical-phonon-mediated scattering event involves a transfer of the energy of an optical phonon. Since this energy is relatively large, conservation of energy dictates that the momentum transfer $\hbar q$ must also be large, which together with the $\exp (-q d)$ form of the interaction implies that the optical-phonon-mediated drag effect is usually small. However, as shown below, under certain experimental circumstances the effect may be relatively large and clearly observable. Provided the substantial experimental hurdles can be surmounted, it may be possible to perform a roomtemperature drag experiment.

The paper is organized as follows. In Sec. II, the formalism for optical-phonon-mediated drag in multiple subband systems is presented. In Sec. III the details of the calculation and the results are presented, both for the case of a single and double subband system. Finally, the conclusions are presented in Sec. IV.

\section{FORMALISM}

In this section, the generalization for the expression for the drag transresistivity for the case of several subbands is given. Then, the explicit form for polar optical-phononmediated interaction is introduced, following the formalism 
for phonon-mediated drag given by Zhang and Takahashi ${ }^{12}$ and Bønsager et al. ${ }^{16}$

\section{A. Transresistivity with multiple subband systems}

In a typical drag experiment, two electrically isolated 2DEGs are placed close together. A current in passed through one layer (denoted layer 1), which induces a voltage in the second (layer 2). The quantity experimentalists measure is the transresistivity $\overleftrightarrow{\rho}_{21}$, which is the ratio of the electric field in the second layer to the current density in the first layer,

$$
\overleftrightarrow{\rho}_{21} \cdot \mathbf{J}_{1}=\mathbf{E}_{2} .
$$

Naturally, the stronger the interlayer coupling, the larger the magnitude of $\rho_{21}$.

We define the plane of the 2DEGs as the $x y$ plane. We use upper case letters for three-dimensional vectors and lower case for vectors in the plane of the 2DEG; e.g., $\mathbf{Q}$ $=\left(\mathbf{q}, Q_{z}\right)$. The expression for the linear-response transresistivity for multiple subband systems, in the semiclassical weak interlayer interaction approximation, comes about from a simple generalization of the derivation in Ref. 15. At temperature $T$, with density $n_{i}$ and charge $e_{i}$ of layer $i$,

$$
\begin{aligned}
\overleftrightarrow{\rho}_{21}= & -\frac{\hbar^{2}}{2 \pi e_{1} e_{2} n_{1} n_{2} k_{B} T} \int \frac{d \mathbf{q}}{(2 \pi)^{2}} \int_{0}^{\infty} d \omega \\
& \times \sum_{\alpha \beta \gamma \delta} \frac{\mathbf{F}_{1}(\alpha \beta ; \mathbf{q}, \omega) \mathbf{F}_{2}(\gamma \delta ; \mathbf{q}, \omega)\left|v_{12}(\alpha \beta, \gamma \delta ; \mathbf{q}, \omega)\right|^{2}}{\sinh ^{2}\left(\hbar \omega / 2 k_{B} T\right)} .
\end{aligned}
$$

Here, $v_{12}(\alpha \beta \gamma \delta ; \mathbf{q}, \omega)$ is the effective interlayer interaction, which scatters carriers from subband $\alpha \rightarrow \beta$ in layer 1 and $\gamma \rightarrow \delta$ in layer 2 , with the change of in-plane momentum $\hbar \mathbf{q}$ and energy $\hbar \omega$ (see Fig. 1). The functions $\mathbf{F}$ are given by

$$
\begin{aligned}
\mathbf{F}(\alpha \beta ; q, \omega)= & \frac{2 \pi e}{\hbar \mu_{t}} \int \frac{d \mathbf{k}}{(2 \pi)^{2}}\left[f_{0 \alpha}(\mathbf{k})-f_{0 \beta}(\mathbf{k}+\mathbf{q})\right] \\
& \times \delta\left[\varepsilon_{\alpha}(\mathbf{k})-\varepsilon_{\beta}(\mathbf{k}+\mathbf{q})-\hbar \omega\right] \\
& \times\left[\mathbf{v}_{\alpha}(\mathbf{k}+\mathbf{q}) \tau_{\alpha}(\mathbf{k}+\mathbf{q})-\mathbf{v}_{\beta}(\mathbf{k}) \tau_{\beta}(\mathbf{k})\right],
\end{aligned}
$$

where $\mu_{t}$ is the mobility of the sample, $\mathbf{v}_{\alpha}$ is the velocity, $\tau_{\alpha}$ is the relaxation time, and $f_{0 \alpha}$ is the Fermi-Dirac distribution function in subband $\alpha$. This relaxation time is defined by the linear response $\delta f$ to a small electric field $\mathbf{E}$,

$$
\delta f_{\alpha}(\mathbf{k})=\left(-\frac{\partial f_{0 \alpha}}{\partial \varepsilon}\right) e_{i} \mathbf{E} \cdot \mathbf{v}_{\alpha}(\mathbf{k}) \tau_{\alpha}(\mathbf{k})
$$

For parabolic isotropic systems where intralayer electronelectron scattering dominates over other scattering mechanisms, the distribution functions in all subbands are drifted Fermi-Diracs. ${ }^{15,18}$ Then, the expression for the transresistivity becomes
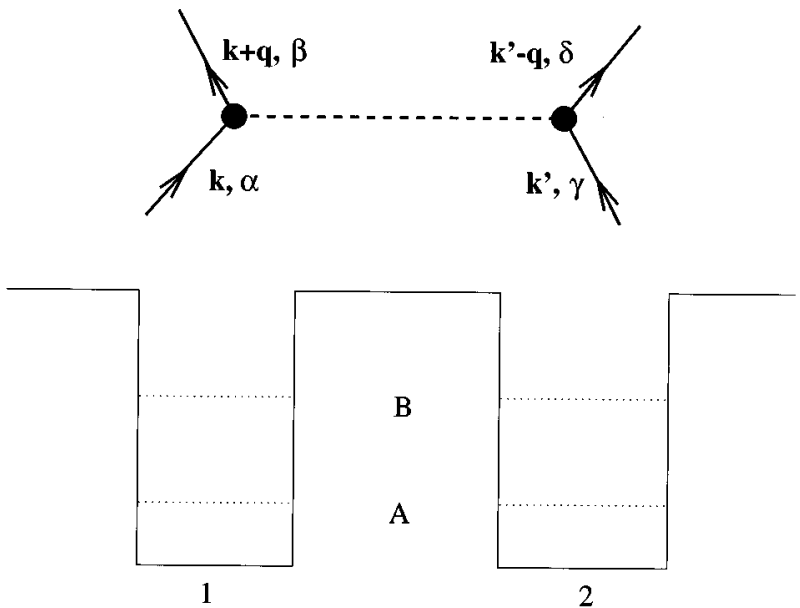

FIG. 1. Schematic for the double-quantum-well system with two subbands, denoted $A$ for lower and $B$ for upper. Experimentally, current is passed through layer 1 and a voltage is measured in layer 2. The phonon-mediated scattering is indicated by the Feynman diagram. The filled dots are the electron-phonon vertices and the dotted line indicates the phonon Green function. The $\alpha \beta \gamma \delta$ are the subband indices, $\mathbf{k}, \mathbf{k}^{\prime}$ are the in-plane momenta before collision, and $\mathbf{q}$ is the in-plane momentum transfer.

$$
\begin{aligned}
\rho_{21}= & -\frac{h}{e^{2}} \frac{1}{4 \pi} \int_{0}^{\infty} \frac{d q q^{3}}{k_{F}^{4}} \int_{0}^{\infty} \frac{\hbar d \omega}{k_{B} T} \\
& \times \sum_{\alpha \beta \gamma \delta} \frac{\chi_{1}^{\prime \prime}(\alpha \beta ; q, \omega) \chi_{2}^{\prime \prime}(\gamma \delta ; q, \omega)\left|v_{12}(\alpha \beta, \gamma \delta ; q, \omega)\right|^{2}}{\sinh ^{2}\left(\hbar \omega / 2 k_{B} T\right)},
\end{aligned}
$$

where $\chi_{i}^{\prime \prime}$ is the imaginary part of the random phase approximation irreducible polarizability

$$
\begin{aligned}
\chi_{i}^{\prime \prime}(\alpha \beta ; q, \omega)= & \int \frac{d \mathbf{k}}{2 \pi}\left[f_{0 \alpha}(\mathbf{k})-f_{0 \beta}(\mathbf{k}+\mathbf{q})\right] \\
& \times \delta\left[\varepsilon_{\alpha}(\mathbf{k})-\varepsilon_{\beta}(\mathbf{k}+\mathbf{q})-\hbar \omega\right] .
\end{aligned}
$$

We shall assume that this form is valid for the remainder of the paper.

\section{B. Interlayer phonon-mediated interaction}

We assume that the properties of the phonon are the same in both the well and the barrier region. We denote the (bulk) electron-phonon interaction matrix element $M_{\lambda}\left(\mathbf{q}, Q_{z}\right)$, where $\lambda$ is the polarization (for clarity the $\lambda$ will henceforth be suppressed).

Let $\psi_{i \alpha}(z)$ be the transverse wave functions of the electrons in well $i$ and subband $\alpha$. A generalization of Refs. 12 and 16 to multiple subbands for an effective bare phonon propagator that scatters electrons from $\alpha \rightarrow \beta$ in well $i$ and $\gamma \rightarrow \delta$ in well $j$ gives

$$
\begin{aligned}
d_{i j}^{(0)}(\alpha \beta, \gamma \delta ; \mathbf{q}, \omega)= & \int_{-\infty}^{\infty} \frac{d Q_{z}}{2 \pi} D^{(0)}\left(\mathbf{q}, Q_{z}, \omega\right)\left|M\left(\mathbf{q}, q_{z}\right)\right|^{2} \\
& \times f_{i}\left(\alpha \beta, Q_{z}\right) f_{j}\left(\gamma \delta,-Q_{z}\right),
\end{aligned}
$$

where $f_{i}\left(\alpha \beta, Q_{z}\right)$ is the form factor given by 


$$
f_{i}\left(\alpha \beta, Q_{z}\right)=\int_{-\infty}^{\infty} d z \psi_{i \alpha}^{*}(z) \psi_{i \beta}(z) e^{-i Q_{z} z}
$$

and $D^{(0)}(\mathbf{Q}, \omega)$ is the bare (retarded) bulk phonon Green function. This effective phonon propagator mediates the interaction between the electrons in the adjacent wells.

\section{Longitudinal polar optical phonons}

The bulk longitudinal polar optical-phonon-electron interaction matrix element is given by ${ }^{19}\left|M^{2}(Q)\right|$ $=V_{c}(Q) \hbar \omega_{\mathrm{LO}}\left(\epsilon_{\infty}^{-1}-\epsilon_{0}^{-1}\right) / 2$, where $V_{c}(Q)=4 \pi e^{2} /\left(\epsilon_{\infty} Q^{2}\right)$ is the bare Coulomb interaction.

We assume that the optical phonons have a constant frequency $\omega_{\mathrm{LO}}$, since the longitudinal optical phonons have a small dispersion at the Brillouin-zone center. Also assuming a finite lifetime $\gamma_{\mathrm{LO}}^{-1}$ gives the form for the bare phonon Green function

$$
D_{\mathrm{LO}}^{(0)}(\omega)=\frac{1}{\omega-\omega_{\mathrm{LO}}+i \gamma_{\mathrm{LO}} / 2}-\frac{1}{\omega+\omega_{\mathrm{LO}}+i \gamma_{\mathrm{LO}} / 2} .
$$

With these assumptions, the phonon Green function $D$ can be factored out of Eq. (7), and the spatial form of the effective longitudinal polar optical-phonon-mediated interaction is exactly the same as the Coulomb interaction. Using the phonon matrix element given above, we obtain

$d_{i j, \mathrm{LO}}(\alpha \beta ; \gamma \delta ; \mathbf{q}, \omega)=D_{\mathrm{LO}}^{(0)}(\omega) \frac{\omega_{\mathrm{LO}}}{2}\left(1-\frac{\epsilon_{\infty}}{\epsilon_{0}}\right) V_{i j}^{c}(\alpha \beta, \gamma \delta ; q)$,

where $V_{i j}^{c}(\alpha \beta, \gamma \delta ; q)$ is the Coulomb matrix element scattering between $\alpha \rightarrow \beta$ in well $i$ and $\gamma \rightarrow \delta$ in well $j$, together with an in-plane momentum change of $\hbar \mathbf{q}$.

\section{Effective screened interlayer interaction}

The optical-phonon contribution to the drag rate is obtained to lowest order by substituting a $d_{12, \mathrm{LO}}^{(0)}$ into $v_{12}$ in Eq. (2) ${ }^{20}$ It has been shown, however, that screening of the interaction produces many novel effects in drag experiments. ${ }^{15,13,21}$ In principle, screening within the random phase approximation can be included for systems where two subbands participate in each layer, but one needs to invert a $16 \times 16$ matrix to find the screened interaction. However, we shall see that screening is not an important consideration in the case of optical-phonon-mediated drag.

One important effect of screening is to remove a spurious mathematical divergence in the transresistivity in Eq. (2). If the phonon lifetime is infinite $(\gamma=0)$, then in the absence of screening the bare phonon interaction gives a nonintegrable divergent momentum transfer at the phonon frequency $\omega_{\mathrm{ph}}(q)$ for all $q$. Even if the lifetime is finite but large, the screening of the electron-phonon interaction is the dominant physical mechanism for elimination of the divergence in the integrand around $\omega_{\mathrm{ph}}(q) .{ }^{16}$ In the case of the acoustic phonons, the lifetime is very large (on the order of a microsecond) because acoustic phonons are unable to decay via anharmonic terms to other phonons, and hence proper treatment of the screening is important to obtain a quantitatively correct result. On the other hand, the optical phonons can decay via anharmonic terms, leading to much shorter intrinsic lifetimes (on the order of picoseconds). In this case, the broadening of the phonon Green function due to the lifetime is the physically dominant mechanism for the removal of the divergence around $\omega_{\mathrm{ph}}(q)$.

Furthermore, as shown below, most of the contribution to the integral Eq. (2) comes from the small $q$ and $\omega \approx \omega_{\mathrm{LO}}$ regime, where the effects of screening are small. Therefore, given the short lifetime of the longitudinal polar optical phonons and the weakness of the screening in the region of $\omega-q$ space relevant for optical phonons, it is justified to use the bare interaction $d^{(0)}$ as the interlayer interaction. ${ }^{22}$

\section{CALCULATION AND RESULTS}

Longitudinal optical phonons are well defined excitations when $\gamma_{\mathrm{LO}}$ is much smaller than $\omega_{\mathrm{LO}}$. Since $\gamma_{\mathrm{LO}}$ is also much smaller than all other frequency scales in the problem, one can approximate

$$
\left|D_{\mathrm{LO}}^{(0)}(\omega)\right|^{2} \approx \frac{2 \pi}{\gamma_{\mathrm{LO}}}\left[\delta\left(\omega-\omega_{\mathrm{LO}}\right)+\delta\left(\omega+\omega_{\mathrm{LO}}\right)\right] .
$$

Note that the real (imaginary) parts of $D_{\mathrm{LO}}^{(0)}(\omega)$ correspond to the virtual (real) phonon contribution. ${ }^{16}$ In the case of optical-phonon-mediated interaction, the virtual and real phonons contribute equally to the drag.

Using Eq. (11) and Eq. (10) in Eq. (5) and the other approximations stated above, the polar optical-phononmediated drag transresistivity is given by

$$
\begin{aligned}
\rho_{21} \approx & \frac{1}{8} \frac{h}{e^{2}}\left(1-\frac{\epsilon_{\infty}}{\epsilon_{0}}\right)^{2} \frac{\omega_{\mathrm{LO}}}{\gamma_{\mathrm{LO}}} \frac{\hbar \omega_{\mathrm{LO}}}{k_{B} T} \frac{1}{\sinh ^{2}\left(\hbar \omega_{\mathrm{LO}} / 2 k_{B} T\right)} \\
& \times \int_{0}^{\infty} d \widetilde{q} \widetilde{q}^{3} \sum_{\alpha \beta \gamma \delta} \chi_{1}^{\prime \prime}\left(\alpha \beta ; \widetilde{q}, \omega_{\mathrm{LO}}\right) \chi_{2}^{\prime \prime}\left(\gamma \delta ; \widetilde{q}, \omega_{\mathrm{LO}}\right) \\
& \times\left|V_{12}^{c}(\alpha \beta, \gamma \delta ; \widetilde{q})\right|^{2}
\end{aligned}
$$

where $\tilde{q}=q / k_{F}$. Note that the apparent divergence of the above result $\gamma_{\mathrm{LO}} \rightarrow 0$ is an artifact of the neglect of the dynamical screening scheme, as discussed in the preceding section.

We now discuss drag in the case when one has single and double subbands in each layer.

\section{A. Drag in single-subband systems}

In the case of drag when there is only a single subband occupied, the expression Eq. (5) reduces to the well-known one given previously. ${ }^{10,23}$ The main difference between previous treatments and optical-phonon-mediated drag is that optical-phonon scattering involves a relatively large exchange of energy, which necessarily implies that the in-plane transfer of momentum is quite large.

In the case when an electron emits or absorbs an optical phonon, its kinetic energy in the direction of the plane changes by $\hbar\left(\omega_{\mathrm{LO}}-\omega_{\alpha \beta}\right)$, where $\omega_{\alpha \beta}$ is the energy change between the subbands $\alpha$ and $\beta$. The momentum transfer $\delta \mathbf{q}$ associated with this for a parabolic band is

$$
\delta \mathbf{q} \cdot\langle\mathbf{v}\rangle=\omega_{\mathrm{LO}}-\omega_{\alpha \beta},
$$


where $\langle\mathbf{v}\rangle$ is the velocity of the average of the initial and final state. In the case where the electron stays within the same subband, $\omega_{\alpha \beta}=0$, and hence $q \gtrsim \omega_{\mathrm{LO}} /\langle v\rangle$.

We insert numbers for GaAs. For density $n=1.5$ $\times 10^{11} \mathrm{~cm}^{-2},\langle v\rangle \approx v_{F} \approx 1.7 \times 10^{7} \mathrm{~cm} / \mathrm{s}$, and $\omega_{\mathrm{LO}} \approx 6 \times 10^{13}$ $\mathrm{s}^{-1}$, one obtains a typical $q$ of approximately $3 \times 10^{6} \mathrm{~cm}^{-1}$. The typical distance separating the wells $d$ is on the order of $400 \AA$, giving a product $q d \approx 14$. Since the Coulomb interaction [and hence by Eq. (10) the optical-phonon-mediated interaction] goes as $\exp (-q d)$, and the scattering strength is given by the matrix element squared, one obtains a factor of $e^{-28}$, which makes it almost impossible to see the effect.

In order to see an effect in single-subband systems, it is clear that one must reduce the typical momentum transfer $\delta \mathbf{q}$. To do this, one must have a lower optical-phonon frequency and a larger $\langle v\rangle$; i.e., a smaller effective mass. A possible candidate is InSb. It has an effective mass of approximately $0.02 m_{e}$, where $m_{e}$ is the bare electron mass, and a longitudinal optical-phonon frequency of $24 \mathrm{meV}$ (compared to $0.067 m_{e}$ and $36 \mathrm{meV}$, respectively, for GaAs). Assuming an electron density $n=1.5 \times 10^{11} \mathrm{~cm}^{-2}$, $d=300 \AA$, and well width $L=200 \AA$, the integral in Eq. (12) for $k_{B} T \lesssim \hbar \omega_{\mathrm{LO}}$ is approximately $1.9 \times 10^{-3}$. The coupling constant $\left(1-\epsilon_{\infty} / \epsilon_{0}\right)^{2}=5 \times 10^{-3}$, yielding

$$
\rho_{21} \approx 30 \mathrm{~m} \Omega \frac{\hbar \omega_{\mathrm{LO}}}{k_{B} T} \frac{1}{\sinh ^{2}\left(\hbar \omega_{\mathrm{LO}} / 2 k_{B} T\right)} \frac{\omega_{\mathrm{LO}}}{\gamma_{\mathrm{LO}}} .
$$

For $k_{B} T=\hbar \omega_{\mathrm{LO}} / 2$, the temperature terms are equal to 1.44. Therefore, we have for the parameters mentioned above

$$
\rho_{\mathrm{InAs}}(T=110 \mathrm{~K}) \approx 40 \mathrm{~m} \Omega \frac{\omega_{\mathrm{LO}}}{\gamma_{\mathrm{LO}}} .
$$

The result depends on the product of the optical-phonon frequency and lifetime. In a relatively clean system, this ratio is large. Using GaAs as a guide, the lifetime $\tau \approx 7 \mathrm{ps}$, at 77 $\mathrm{K}^{24}$ Therefore, $\gamma_{\mathrm{LO}}=6.6 \times 10^{-16} \mathrm{eV} / 5 \times 10^{-12} \approx 0.1 \mathrm{meV}$, giving $\omega_{\mathrm{LO}} / \gamma_{\mathrm{LO}} \approx 360$. This leaves one with a transresistivity on the order of 1-10 $\Omega$, which should be easily measurable. As a comparison, Gramila et al. ${ }^{4}$ could measure transresistivities down to approximately $1 \mathrm{~m} \Omega$, despite lowtemperature conditions that required drive currents to be kept down at $200 \mathrm{nA}$ to avoid heating the sample.

\section{B. Double-subband systems}

We define the lower subband as $A$ and upper subband as $B$. In double-subband systems, one can tune $\omega_{B A}$ so that it is equal to $\omega_{\mathrm{LO}}$. Then, from Eq. (13) the minimum momentum transfer is zero, and therefore there can be a significant contribution from small in-plane momentum transfer events, without the cutoff in the exponential in $V_{21}^{c}(q)$. As a consequence, we shall see that the drag rate can be rather large.

We assume the wells are made of GaAs. As mentioned above, the intrasubband excitations do not give significant contributions. Therefore, the main contributions come from the terms $\chi_{i}^{\prime \prime}(A B ; q, \omega)$. Since the integral in Eq. (5) is restricted to $\omega>0$, the only term in the sum over the subband

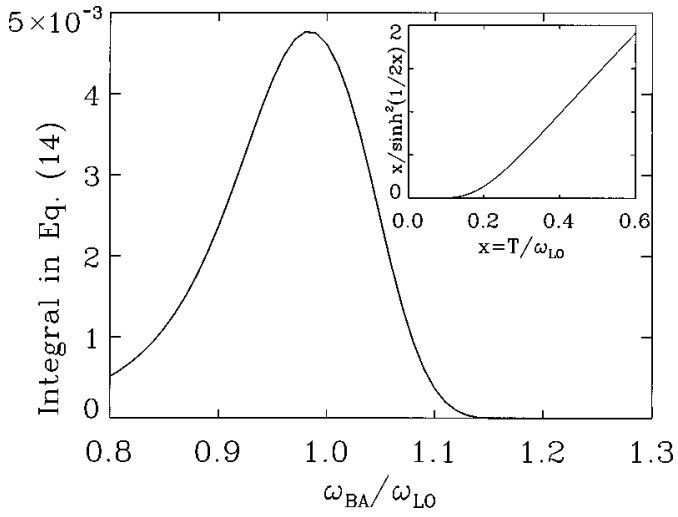

FIG. 2. The integral in Eq. (12), $d=400 \AA, \quad n=1.5$ $\times 10^{11} \mathrm{~cm}^{-2}$, and $\omega_{\mathrm{LO}}=36 \mathrm{meV} \approx 400 \mathrm{~K}$, as in GaAs. We assume infinite square wells for the confinement, in which case $\omega_{A B}$ $=\omega_{\mathrm{LO}}$ for $L \approx 215 \AA$. Inset: temperature dependence of the transresistivity for low temperatures, $x / \sinh ^{2}(1 / 2 x)$ as a function of $x$ $=\hbar \omega_{\mathrm{LO}} / k_{B} T$ [see Eqs. (12) and (14)].

indices that contributes significantly for $k_{B} T \lesssim \hbar \omega_{\mathrm{LO}}$ is $\chi_{1}(A B ; q, \omega) \chi_{2}(A B ; q, \omega)$. In this case, using a square well potential, we have

$$
\begin{aligned}
V_{12}^{c}(A B, A B ; q)= & \frac{2 \pi e^{2}}{\epsilon_{\infty} q} \exp (-q d) \\
& \times\left[\frac{16 \cosh (q L / 2) \pi^{2} q L}{9 \pi^{2}+10 \pi^{2}(q L)^{2}+(q L)^{4}}\right]^{2} .
\end{aligned}
$$

Using the above in Eq. (12) allows us to calculate $\rho_{21}$. For GaAs, the term $h /\left(8 e^{2}\right)\left(1-\epsilon_{\infty} / \epsilon_{0}\right)^{2} \approx 65 \Omega$. For temperatures $k_{B} T \lesssim \hbar \omega_{\mathrm{LO}}$ at typical experimental densities, the term in the integrand in Eq. (12) is not a strong function of temperature and hence can be evaluated in the $T=0$ limit [when $k_{B} T \geqslant \hbar \omega_{\mathrm{LO}}$, the lowest subband becomes nondegenerate and a more careful evaluation of the temperature dependence of $\chi_{i}^{\prime \prime}$ in Eq. (12) is necessary]. Figure 2 shows this integral in Eq. (12) as a function of the energy difference $\omega_{A B}$. The drag rate peaks as $\omega_{A B}=\omega_{\mathrm{LO}}$ for the reasons stated above. Estimating the ratio $\omega_{\mathrm{LO}} / \gamma_{\mathrm{LO}} \approx 200,{ }^{24}$ we see that at $T=100 \mathrm{~K}$ the optical-phonon-mediated drag is on the order of $30 \Omega$. As the subband separation moves further away from $\omega_{\mathrm{LO}}$, the transresistivity decreases rapidly. The optical-phonon-mediated interaction is unique in that it is particularly sensitive to the width of the wells.

Lastly, note that unlike the single-subband case, doublesubband InSb quantum wells will not give substantially larger drag rates than their GaAs counterparts because the magnitude of the drag is not limited by a minimum $q$ transfer. Thus, the magnitude of $\rho_{21}$ for both GaAs and $\mathrm{InSb}$ should be roughly the same.

\section{CONCLUSIONS}

We have calculated the transresistivity for coupled quantum wells due the mediation of polar optical phonons. The spatial form of the polar optical phonon is identical to that of the Coulomb interaction. We find that in GaAs, when only a single subband is important (i.e., $\hbar \omega_{A B} \gg E_{F}, k_{B} T$ ), the transresistivity is negligible at present experimental condi- 
tions. This is because the polar optical-phonon scattering is an inelastic event resulting in a minimum $q$-vector, which substantially reduces the effect. However, it may be possible to see single-subband drag in other materials with lower $\omega_{\mathrm{LO}}$ and lower effective masses, such as InSb. In the case where there are two different subbands, the subband energy difference can be engineered to be the same as the $\omega_{\mathrm{LO}}$, in which case the optical-phonon-mediated transresistivity can be relatively large and observable. The magnitude of the drag should be sensitive to the subband energy separation relative to the optical-phonon frequencies. It may be possible to observe this resonance by tuning the subband energy splitting through $\omega_{\text {LO }}$ by means of an external gate, which changes the shape of the confining potential in the wells.

In this work we have assumed that the intralayer electron- electron interactions are strong enough that the effective $\tau$ is constant (i.e., a drifted Fermi-Dirac distribution for all subbands). For the clean samples used in these experiments, this is generally the case. We have also assumed that the phonon frequencies in the barrier and in the well are identical. In fact, the optical phonons in $\mathrm{Al}_{x} \mathrm{Ga}_{1-x} \mathrm{As}$ have frequencies that are, depending on $x$, somewhat higher than GaAs. The peak shown in Fig. 2 would tend to be smeared by this mismatch in frequencies.

\section{ACKNOWLEDGMENTS}

The author thanks M. C. Bønsager, K. Flensberg, T. J. Gramila, A.-P. Jauho, and A. H. MacDonald for useful discussions and comments.
${ }^{1}$ M. B. Pogrebinskii, Fiz. Tekh. Poluprovodn. 11, 637 (1977) [Sov. Phys. Semicond. 11, 372 (1977)].

${ }^{2}$ P. J. Price, Physica B 117, 750 (1983).

${ }^{3}$ P. M. Solomon, P. J. Price, D. J. Frank, and D. C. La Tulipe, Phys. Rev. Lett. 63, 2508 (1989).

${ }^{4}$ T. J. Gramila, J. P. Eisenstein, A. H. MacDonald, L. N. Pfeiffer, and K. W. West, Phys. Rev. Lett. 66, 1216 (1991); Surf. Sci. 263, 446 (1992); Phys. Rev. B 47, 12957 (1993); Physica B 197, 442 (1994).

${ }^{5}$ U. Sivan, P. M. Solomon, and H. Shtrikman, Phys. Rev. Lett. 68, 1196 (1992).

${ }^{6}$ H. Rubel, E. H. Lindfield, D. A. Ritchie, K. M. Brown, M. Pepper, and G. A. C. Jones, Semicond. Sci. Technol. 10, 1229 (1995); Surf. Sci. 361/362, 134 (1996).

${ }^{7}$ H. Rubel, A. Fischer, W. Dietsche, K. von Klitzing, and K. Eberl, Phys. Rev. Lett. 78, 1763 (1997).

${ }^{8}$ N. P. R. Hill, J. T. Nicholls, E. H. Linfield, M. Pepper, D. A. Ritchie, A. R. Hamilton, and G. A. C. Jones, J. Phys.: Condens. Matter 8, L557 (1996).

${ }^{9}$ N. P. R. Hill, J. T. Nicholls, E. H. Linfield, M. Pepper, D. A. Ritchie, G. A. C. Jones, B. Y.-K. Hu, and K. Flensberg, Phys. Rev. Lett. 78, 2204 (1997).

${ }^{10}$ A.-P. Jauho and H. Smith, Phys. Rev. B 47, 4420 (1993).

${ }^{11}$ H. C. Tso, P. Vasilopoulos, and F. M. Peeters, Phys. Rev. Lett. 68, 2516 (1992).

${ }^{12}$ C. Zhang and Y. Takahashi, J. Phys.: Condens. Matter 5, 5009 (1993).

${ }^{13}$ K. Flensberg and B. Y.-K. Hu, Phys. Rev. Lett. 73, 3572 (1994).

${ }^{14}$ L. Sẃierkowski, J. Szymanśki, and Z. W. Gortel, Phys. Rev. Lett. 74, 3245 (1995).
${ }^{15}$ K. Flensberg and B. Y.-K. Hu, Phys. Rev. B 52, 14796 (1995).

${ }^{16}$ M. C. Bønsager, K. Flensberg, B. Y.-K. Hu, and A. H. MacDonald, Phys. Rev. B 57, 7085 (1998).

${ }^{17}$ K. Güven and B. Tanatar, Phys. Rev. B 56, 7535 (1997).

${ }^{18}$ B. Y.-K. Hu and K. Flensberg, Phys. Rev. B 53, 10072 (1996).

${ }^{19}$ See, e.g., G. D. Mahan, Many-Particle Physics, 2nd ed. (Plenum Press, New York, 1990).

${ }^{20}$ In principle, the bare interlayer interaction $v_{12}$ is the sum of the Coulomb term $V_{12}^{c}$ and phonon term $d$, and there are interference terms between these two terms. However, the optical-phonon contribution is strong and sharp and localized in $q-\omega$ space, and hence the interference effects $V_{12}^{c} d$ are minimal compared to the "purely phonon"' term $d^{2}$.

${ }^{21}$ M. C. Bønsager, K. Flensberg, B. Y.-K. Hu, and A.-P. Jauho, Phys. Rev. Lett. 77, 1366 (1996).

${ }^{22}$ One possible screening effect that might complicate the situation is coupling the optical phonons to intersubband plasmons [A. Kuznetsov (private communication)]. As the intersubband plasmons always lie above the intersubband single-particle continuum due to the depolarization shift, they do not directly participate in the drag, but they can couple to the optical phonons and shift the frequency of that mode. The main qualitative effects mentioned in this paper, however, should not be altered.

${ }^{23}$ L. Zheng and A. H. MacDonald, Phys. Rev. B 48, 8203 (1993).

${ }^{24}$ B. K. Ridley, J. Phys.: Condens. Matter 8, 2511 (1996); E. HaroPoniatowski, J. L. Escamilla-Reyes, and K. H. Wanser, Phys. Rev. B 53, 12121 (1996); A. R. Bhatt, K. W. Kim, and M. A. Stroscio, J. Appl. Phys. 76, 3905 (1994). 